\title{
Beyond recruitment: good participatory practice enhances the impact of research in a pandemic
}

In a health emergency, clear, two-way communication between researchers and a broad spectrum of stakeholders is essential to establishing trust-a prerequisite for meaningful uptake of new treatments and vaccines.

\section{Barthalomew Wilson, Katharine Wright, Robert Taylor and Elizabeth Higgs}

T ust is crucial to the medical research enterprise. Absence of trust in research and researchers-whether among the medical establishment, potential participants, or the wider public-can disrupt and delay the conduct of studies. Of greater consequence in a pandemic, it can have a negative effect on the public acceptability and, hence, the uptake of research results, including the new treatments and vaccines needed to respond effectively to novel pathogens. Lack of trust, in other words, risks undermining the fundamental rationale for undertaking research in the first place.

In the case of a global pandemic such as COVID-19, lack of trust has consequencesfor the people who refuse novel interventions because they lack confidence in the process by which they have been developed, and for everyone, in prolonging the enormous disruption to normal life engendered by the pandemic. In fact, the first goal of the new US COVID-19 strategy is to "restore trust with the American people"

\section{Building trust}

How is trust created-especially in the stressful, time-pressed, and fearful climate of a global pandemic? Much has been written in recent years about the need for 'trustworthiness' on the part of those asking others to trust them ${ }^{2}$. Trust is not the default condition, and it is even harder to build without a solid foundation of trust before a crisis hits. It is something that researchers must earn over time through the attitudes, values, and practices that they bring to their work.

A core element in creating environments of trust is found in 'good participatory practice' (GPP) that allows all stakeholders-a broad spectrum that includes both those able to affect the conduct of the research and those liable to be affected by it-to contribute to accomplishing a trial's research aims. With roots in community engagement for research into human immunodeficiency virus in the $1980 \mathrm{~s}^{3}$ and subsequently

\section{Good participatory practice: layers of trial stakeholders}
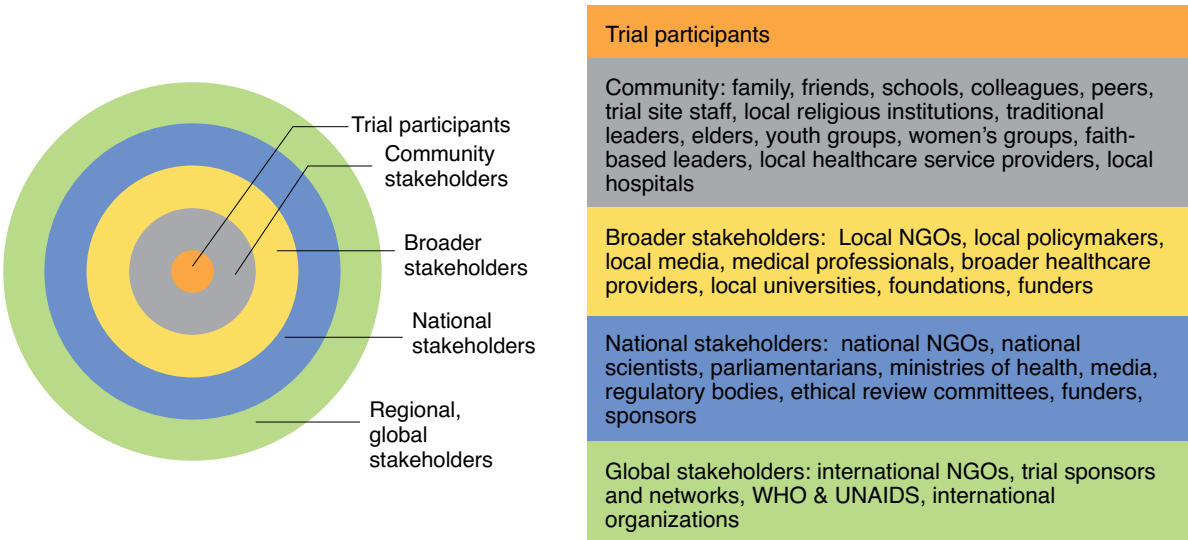

Fig. 1 | The multiple layers of stakeholders in clinical research; researchers tend to pay too much attention to the two outer circles and not enough to the three inner circles. NGOs, non-governmental organizations; WHO, World Health Organization; UNAIDS, Joint United Nations Programme on HIV/AIDS. Adapted from ref. ${ }^{4}$.

developed further ${ }^{4-6}$, these practices allow researchers to bring in all trial stakeholders as essential members of the research team, which makes research a genuinely joint enterprise.

Implementing GPP requires effort, resources, and expertise, and the list of stakeholders is both multi-tiered and long (Fig. 1). It is nonetheless essential, on both moral and practical grounds. The moral case for working in respectful partnership with stakeholders has been made elsewhere ${ }^{7}$. In practical terms, if a substantial part of a population does not trust that a trial is scientifically rigorous and is conducted by people working for the public good, the trial can grind to a halt amid rumors and recriminations; even if the trial is completed, the research results might simply be ignored. Sometimes distrust is rooted in a grim history of oppression and betrayal ${ }^{8}$. Sometimes it is just the result of the latest social-media rumor $^{9}$. Either way, the solution is for those who plan, fund, and conduct clinical research to see the broad group of stakeholders not as a set of disparate communities to be placated, but as integral parts of the research team.

\section{GPP in practice}

What does this mean in practice? Below we provide three examples to illustrate the role GPP can play in clinical research, and give some high-level, pragmatic advice about how to implement GPP in clinical research.

Ebola trials in Liberia. When the USA and Liberia forged the Partnership for Research on Ebola Virus in Liberia (PREVAIL) in October 2014, to confront the West African Ebola outbreak, the environment for clinical research in Liberia was extraordinarily difficult. The very name of the disease evoked enormous fear, rumors were rife, and trust in researchers-both foreign and domestic-was almost nonexistent. Community and national leaders were not at all inclined to support the conduct of research on human subjects in the midst of a disastrous epidemic.

However, PREVAIL launched its first vaccine trial in February 2015 and brought it to a successful conclusion. That and 
other PREVAIL trials would not have been possible without the Liberian-led social mobilization, community engagement, and communication (SMC) team that developed and executed a comprehensive roadmap to bring stakeholders into the trial. The SMC team convinced key leaders to allow the trial to begin, maintained bidirectional communication channels with affected communities and stakeholders, actively identified problems and rumors, corrected misinformation, and conducted media campaigns and other innovative outreach efforts.

Many factors contributed to that success. In the broadest terms, PREVAIL was conceived from the beginning as a genuine partnership. Some initial distrust flowed from a lingering history of foreign exploitation, corruption, and lack of transparency and accountability. It therefore mattered that Liberian doctors, government officials, and community members were not just 'informed' about PREVAIL but actually became an integral part of the research team-they no longer were outsiders being asked to trust a group of foreign researchers. The SMC team then forged and strengthened more partnerships with a broad range of stakeholders, including communities, to change negative perceptions in the broader population into positive relationships by engaging local expertise.

Two more specific examples illustrate the SMC team's activities. First, early listening sessions with community members revealed that the word 'trial'- which the local community thought of as an experiment done on animals-should be replaced with the more acceptable 'study'. This was a small point, but an important one that could have easily been missed without the listening phase.

Second, on the basis of recommendations from community leaders, 'trackers' were nominated by the local community to join the study team to support study participants. These trackers were instrumental in achieving an enviable follow-up rate of more than $98 \%$. Equally importantly, they also became local community liaisons, educating the wider community about the trial and communicating problems back to the SMC team. For example, they were able to alert the study team to the stigma faced by trial participants that in some cases led to their being evicted from their homes, and were able to inform the study team about circulating rumors and misinformation. The SMC team then addressed these problems with targeted messaging and broader sharing of trial information through community meetings and radio spots, including jingles from the nationally recognized traditional leader Queen Juli Endee (https://www. youtube.com/watch? $\mathrm{v}=\mathrm{tHbLDsXX20w)}$.

The RECOVERY trial in the UK. The UK Randomized Evaluation of COVID-19 Therapy (RECOVERY) trial recruited 12,000 participants from 176 different hospitals in its first three months ${ }^{10}$. Through a multi-arm adaptive design, it identified dexamethasone (a widely used anti-inflammatory steroid) as an effective treatment for COVID-19 in patients requiring oxygen or ventilation, and it produced promising provisional results for the monoclonal antibody tocilizumab (already licensed for rheumatoid arthritis) in patients in the intensive care unit. New national guidance in the UK now calls for eligible patients to routinely be offered dexamethasone ${ }^{11}$ and to be considered for tocilizumab ${ }^{12}$. Equally importantly, the RECOVERY trial showed that other candidate therapeutics, such as convalescent plasma, are not effective in treating COVID-19.

Several factors contributed to the rapid deployment, enrollment, and uptake of results for the RECOVERY trial, including pre-existing integration of clinical-research infrastructure with clinical services provided through the National Health Service. National political leaders and medical authorities publicly supported participation in clinical trials, and that support was then widely reported through the media. Other key stakeholders were also engaged early in the process, including the Health Research Authority, which facilitated rapid processes for ethical review and approvals by the National Health Service. Because the full array of stakeholders had been engaged appropriately from the beginning, the study was approved quickly and was given priority for implementation so that enrollment went smoothly. Notably, when new treatment guidelines appeared based on the research, they were efficiently communicated, and immediately changed practice.

\section{Monoclonal antibody trials in the}

USA. Not engaging stakeholders from the outset can lead to public confusion, underutilization of the countermeasures studied, and other problems. For example, surprising results from industry-sponsored phase 1 dose-escalation trials conducted in the USA showed that the monoclonal antibody bamlanivimab (LY-CoV555) reduced hospitalizations and visits to the emergency department due to COVID-19, and that REGN-COV2, a combination of two monoclonal antibodies, reduced medically attended visits. But those positive results were so unexpected that the emergency use authorizations that followed ${ }^{13,14}$ took patients, care providers, and other important stakeholders by surprise. From the pandemic's earliest days, the public-health message for people diagnosed with COVID-19 has been to isolate at home, and to not seek further treatment until symptoms become severe. Without messaging to prepare the way, it was difficult for people to understand that the option to seek out a new effective outpatient treatment might be open to them. Moreover, hospitals and other medical facilities were not at all ready to safely administer the requisite lengthy infusions to a large group of infectious patients. The result has been that in the USA, these drugs are currently underutilized. That problem might have been prevented had a phase $2 / 3$ trial been completed, as had been planned in the US COVID-19 research agenda Accelerating COVID-19 Therapeutic Interventions and Vaccines (led by the US National Institutes of Health) ${ }^{15}$, which included robust GPP efforts such as Rise Above COVID ${ }^{16}$, Community Engagement Alliance Against COVID Disparities ${ }^{17}$, and Combat COVID ${ }^{18}$.

\section{Implementing GPP: a pragmatic overview}

To bring stakeholders into the trial process from the beginning, we suggest that those responsible for planning and funding trials take three early steps to implement GPP. First, identify all stakeholders. Second, make a detailed roadmap for how GPP will be integrated into the trial from the design phase onward to engage those stakeholders. Third, identify specific people to develop and implement the roadmap, making sure they have the operational authority and budget to do so.

The roadmap itself can be separated into three phases covering the life of the trial. Below, we highlight key aspects of the roadmap that relate to engagement with community stakeholders and participants. Other parts of the roadmap will need to include plans to engage with the other regional and national stakeholders described above. Building trust with a diverse group of stakeholders is not an event but a process that requires extensive dialog, transparency, time, and two-way communication.

During trial design. The over-riding purpose of many early GPP activities is to listen and learn, to inform development and implementation of the protocol. Among other tasks, planners must map community groups and identify trusted formal and informal leaders; identify trusted sources of information; identify community beliefs 
and attitudes about clinical research; map the communication channels through which people receive and act on information; and understand the target population's major health concerns, which may not be the disease the trial is about.

A second set of pre-enrollment activities aims to open clear, two-way communication channels with various community groups and other stakeholders. Some of these channels are formal; for example, a community advisory board, designed to provide information and feedback from various groups within the community, should be created with defined procedures governing membership and responsibilities. Some are informal, such as opening informal dialog with leaders of subgroups within a population. Some will rely on mass communication with the population as a whole or with specific target audiences. To use these channels effectively, the study team should develop culturally adapted messages and materials to support the trial, with input from appropriate stakeholders.

During the trial. Once active recruitment and follow-up begins, the research team needs to continue to fully engage stakeholders and maintain a dialog about study activities, using the bi-directional communication channels already established. Local people should be recruited to serve as communicators, mobilizers, and study-participant trackers. The community advisory board should get regular updates from study leadershipand the study team must ensure that feedback from the community is in fact used to guide trial conduct and address new or ongoing problems. Social analytics, which continuously brings together data from multiple communication channels, is becoming increasingly helpful in discerning population attitudes, knowledge, and rumors, particularly during large outbreaks and pandemics ${ }^{19}$.
All the while, a monitoring and evaluation mechanism for community-participation activities should be established and implemented, and the findings should be used to revise GPP strategies.

Closing the trial. How the trial ends can have a major impact on the degree to which people will accept the intervention under study, and on prospects for future research in the same community. The key outcomes must be carefully communicated to different stakeholders, using various media, in order to maximize access to new interventions and implementation of study findings. If healthcare providers are to incorporate any new interventions into standard practice, the necessary resourcesfinancial, human, and infrastructure-must be in place.

\section{GPP from the start}

In a pandemic, it might be tempting to imagine that there is simply no time to consult a wide group of stakeholders. That would be a mistake. Minimizing deaths and accelerating the end of the pandemic is the goal of emergency-response research. But the trust that flows from full implementation of GPP is essential to the successful development of new interventions that will actually be used by the target populations. In short, beginning with the end of the pandemic in mind, one must begin with GPP.

\section{Barthalomew Wilson', Katharine Wright ${ }^{2}$,} Robert Taylor ${ }^{3}$ and Elizabeth Higgs ${ }^{4 凶}$ ${ }^{1}$ Partnership for Research on Vaccines and Infectious Diseases in Liberia, Monrovia, Liberia. ${ }^{2}$ Nuffield Council on Bioethics, London, UK. ${ }^{3}$ National Institute for Allergy and Infectious Diseases, National Institutes of Health (Contractor), Rockville, MD, USA. ${ }^{4}$ National Institute of Allergy and Infectious Diseases, National Institutes of Health, Rockville, $M D$, USA.

凶e-mail: ehiggs@niaid.nih.gov
Published online: 15 March 2021

https://doi.org/10.1038/s41591-021-01271-3

\section{References}

1. US Government. The White House https://www.whitehouse.gov/ wp-content/uploads/2021/01/National-Strategy-for-the-COVID-19 Response-and-Pandemic-Preparedness.pdf (2021).

O'Neill, O. Int. J. Philos. Stud. 26, 293-300 (2018).

3. Fauci, A. S. Acad. Med. 64, 115-119 (1989).

4. The Joint United Nations Programme on HIV/AIDS. UNAIDS https://www.unaids.org/en/resources/documents/2011/20110629 JC1853_GPP_Guidelines_2011\%20OK (2011).

5. Hankins, C. World Health Organization https://www.who.int/ blueprint/what/norms-standards/GPP-EPP-December2016.pdf (2016).

6. World Health Organization. https://www.who.int/docs/defaultsource/science-division/research/blueprint-good-participatorypractice-for-covid-19-clinical-trials---a-toolbox.pdf (2020).

7. Wright, K. \& Parker, M. Nat. Med. 26, 308-309 (2020).

8. Washington, H.A. Medical Apartheid: The Dark History of Medical Experimentation on Black Americans from Colonial Times to the Present (Doubleday, New York, 2006).

9. Neil, S. J. D. \& Campbell, E. M. AIDS Res. Hum. Retroviruses 36, 545-549 (2020).

10. National Institute for Health Research. https://www.nihr.ac.uk case-studies/the-uks-flagship-covid-19-treatment-trial-the-rec overy-trial/25358 (2020).

11. NHS England. https://www.england.nhs.uk/publication/ covid-19-therapy-corticosteroids-including-dexamethasoneand-hydrocortisone/ (2020).

12. Department of Health and Social Care, The Scottish Government, Welsh Government \& NI Department of Health. https://www. cas.mhra.gov.uk/ViewandAcknowledgment/ViewAttachment. aspx?Attachment_id=103745 (2021).

13. U.S. Food \& Drug Administration. https://www.fda.gov/ news-events/press-announcements/coronavirus-covid19-update-fda-authorizes-monoclonal-ant ibody-treatment-covid-19 (9 November 2020).

14. U.S. Food \& Drug Administration. https://www.fda.gov/newsevents/press-announcements/coronavirus-covid-19-updatefda-authorizes-monoclonal-antibodies-treatment-covid-19 (21 November 2020).

15. National Institutes of Health. https://www.nih.gov/researchtraining/medical-research-initiatives/activ (2020).

6. Rise Above Covid. https://www.riseabovecovid.org/en/ (2020).

17. National Institutes of Health Community Engagement Alliance. https://covid19community.nih.gov/ (2020).

18. U.S. Department of Health and Human Services. https://combatcovid.hhs.gov/ (2020).

19. Hall, W., Jamieson, A. \& Wardle, G. https://wellcome.org/sites default/files/advancing-epidemics-rd-2019.pdf (Wellcome Trust, 2019).

\section{Acknowledgements}

For E.H. and R.T.: the opinions expressed are the authors' own and do not necessarily represent the views of the National Institutes of Health.

The authors declare no competing interests. 